\title{
Goal-specific influences on the representation of spatial perspective
}

\author{
HOLLY A. TAYLOR, SUSAN J. NAYLOR, and NICHOLAS A. CHECHILE \\ Thufts University, Medford, Massachusetts
}

\begin{abstract}
In two experiments, subjects learned an unfamiliar campus environment, either by studying a map or by navigating. During acquisition, the subjects had one of two spatial goals: to learn the layout of the building (survey goal) or to learn the fastest routes between locations (route goal). Spatial memory was tested with several tasks, some assessing survey perspective processing and some assessing route perspective processing. Results indicate multiple influences on the representation of spatial perspective. Learning condition influenced performance. Individuals studying maps gave more accurate responses to some survey perspective tasks, whereas individuals navigating gave more accurate responses to some route perspective tasks. Spatial goals also influenced performance. Having a route goal enhanced performance on route perspective tasks; having a survey goal enhanced performance on survey perspective tasks. These findings are discussed in the context of research indicating flexibility when processing spatial perspective. Individuals can use spatial information from different perspectives, often doing so in a goal-directed manner.
\end{abstract}

Negotiations of our environment, such as walking across campus, riding a bike home, or driving to a new destination, require complex spatial information. This information can come from different sources-including exploration, maps, and verbal descriptions - and can be isomorphic, but the sources differ in ways that may impact how we mentally represent the information. Primary differences between spatial information sources fall into two categories: the symbolic nature of the information and the spatial perspective imparted, either route or survey. We will concentrate on the latter. The purpose of the present research is to examine the influence of perspective-based goals (survey and route) on spatial memory from different information sources (maps and navigation). These effects will be examined, using a number of different spatial tasks.

Maps and navigation impart different types of spatial information, most notably the spatial perspective. Perspective generally reflects the viewpoint taken on the environment, either within (route perspective) or above (survey perspective). Other types of knowledge gained through maps and navigation contribute to the overall sense of each perspective, including the reference system for locating new landmarks, whether the orientation is stable or changing, and the amount of information available at a given time. Exploration of an environment provides route information, the characteristics of which include a within-environment viewpoint, a viewer-centered

This research was supported in part by a grant from the Faculty Research Awards Committee at Tufts University. We thank Sal Soraci for helpful discussions of this manuscript and $\mathrm{H}$. Lee Rosenberg for assistance in collecting data. Correspondence concerning this article should be addressed to $\mathrm{H}$. A. Taylor, Research Building, 490 Boston Ave., Tufts University, Medford, MA 02155 (e-mail: htaylor@emerald. tufts.edu). reference system, a changing orientation with each turn taken in the environment, and the limitation of information by the visual field. Maps present survey information, characterized by a bird's-eye viewpoint, an objectcentered reference system, a stable orientation, and the significant availability of information (see Table 1 for a summary). Although these properties are generally true of knowledge gained from each perspective, they are not absolutes. For example, maps are generally kept in one orientation, but are sometimes rotated to be aligned with the environment.

Does the spatial perspective available during learning influence the perspective in memory? A number of studies have found a correspondence between source perspective and memory perspective (Evans \& Pezdek, 1980; Leiser, Tzelgov, \& Henik, 1987; Perrig \& Kintsch, 1985; Sholl, 1987; Thorndyke \& Hayes-Roth, 1982). Evans and Pezdek (1980) and Sholl (1987) have shown that representations derived from maps are orientation specific, whereas those resulting from navigation are not. Maps are generally kept in a single orientation, whereas orientation changes constantly during navigation. Furthermore, Thorndyke and Hayes-Roth found that individuals studying a map had greater knowledge of straight-line distances and relative location, whereas individuals learning by navigation had greater route distance or orientation knowledge. Similar results have been found for spatial perspectives presented through text. Perrig and Kintsch found that route and survey descriptions of an environment led to perspective-consistent representations, particularly for female subjects. Sholl's (1992) spatial memory model suggests that individuals access spatial information with coordinate systems that correspond to the information sources. Map learning leads to an object-centered system, whereas navigation leads to a body-centered system. 
Table 1

Dimensions of Spatial Knowledge

\begin{tabular}{lll}
\hline & \multicolumn{2}{c}{ Knowledge Types } \\
\cline { 2 - 3 } & \multicolumn{1}{c}{ Survey } & \multicolumn{1}{c}{ Route } \\
Viewpoint & above & within \\
Reference system & object centered & viewing centered \\
Orientation & stable & changing \\
Information availability & unlimited & limited \\
\hline
\end{tabular}

Note-Stable orientation is generally true of survey knowledge, but is not absolute. Individuals can rotate a map while learning. Unlimited refers only to the scope of the environment depicted.

Other studies have failed to find representation differences as a function of perspective. McNamara, Hardy, and Hirtle (1989) found spatial priming effects within regions for both map- and navigation-learned environments. Taylor and Tversky (1992b), in four experiments, found that subjects could make spatial inferences in either perspective with equal speed and accuracy, regardless of the learned perspective.

Still other studies indicate that spatial representations change with experience (Thorndyke \& Hayes-Roth, 1982). Golledge and Spector (1978) found that individuals' mental maps of heavily traveled areas were more integrated than those for less traveled areas, indicating a transition to a more configural representation. Siegel and White's (1975) theory of spatial knowledge development predicts this change. In this theory, when individuals learn through navigation, they first acquire landmark information. Second, landmark information is linked in interconnected routes. Finally, routes are integrated, and Euclidean knowledge emerges. Although primarily a model of development, these stages are also suggested for adults learning new environments. Computer models by both Kuipers (1978) and Leiser and Zilbershatz (1989) support this model, predicting that survey representations emerge as a result of additional route experience. Whether these knowledge types develop sequentially (Evans, Marrero, \& Butler, 1981; Leiser et al., 1987; Perrig \& Kintsch, 1985; Siegel \& White, 1975) or simultaneously (McDonald \& Pellegrino, 1993) remains in question.

As the previous discussion illustrates, whether perspective is maintained in memory remains unclear. Several explanations could account for the differences discussed above. First, the measures used to assess spatial knowledge addressed different types of knowledge available through the information sources, such as distance, orientation, relative location, and configuration knowledge. The use of different dependent measures across the studies makes comparison difficult, as individual tasks may have differential reliance on perspective information. On the basis of this possibility, the present studies employed a variety of tasks that have been used previously to assess route and survey perspective processing.

Second, the environments used in these studies varied significantly. In some cases, different environments were used for map and navigation learning (e.g., Sholl, 1987).
This could be problematic for a number of reasons. Taylor and Tversky (1996) found that different features of environments, particularly the number of available paths and the relative size of landmarks, influenced the use of perspective when writing spatial descriptions. In addition, for reasons of cognitive economy, spatial information is often cognitively preprocessed in the form of grouping and/or regularization. Stevens and Coupe (1978) suggest that errors such as believing that Reno, Nevada, is east of San Diego, California, indicate a hierarchical grouping of spatial information. Hierarchical grouping occurs with different types of boundaries (countries, states, counties, and route segments) and even with environments void of natural groupings (McNamara, 1986; McNamara et al., 1989). Regularization includes alignment and rotation (B. Tversky, 1981). We remember environments with features more aligned, with features rotated within a larger contextual framework, and with irregular intersections remembered as being perpendicular (B. Tversky, 1981). Perceptual factors alone do not account for these effects, as memory distortions for visual displays differ, depending on whether the display is interpreted as being a map or a graph (B. Tversky \& Schiano, 1989). In summary, because environmental features are subject to preprocessing and contribute to perspective selection, comparisons between studies with different environments are difficult. The present studies use the same environment for all the experimental conditions.

Third, learning goals may guide how spatial information is processed. We often switch perspectives, depending on our goal for using spatial information. For example, we use maps to find routes, or we draw maps of areas learned by navigation, such as those given to dinner party guests. As in transfer appropriate processing (see, e.g., Morris, Bransford, \& Franks, 1977), the task used during learning has the greatest impact when it matches the retrieval task. In other words, the learning goal ties directly into the circumstances of retrieval.

Goals have different influences on learning. Goals guide attention during learning, serving as anchors for selecting relevant stimuli (Britton, Meyer, Simpson, Holdredge, \& Curry, 1979; LaBerge, 1995; Meyer, 1975). Goals also instantiate schemas for interpreting new information. Pichert and Anderson (1977) instructed students to take one of two perspectives when reading a story, either that of a home buyer or that of a burglar. Ideas consistent with the assigned perspective were better remembered. Finally, goals can serve as retrieval cues. Anderson and Pichert (1978) again had subjects take one of two perspectives in a story. Later, when asked to recall the story from the other perspective, the subjects recalled ideas important to the new perspective and left out information relevant to the original perspective.

Few studies have assessed the influence of goals on spatial memory. Gauvain and Rogoff (1986) told children to learn either the best route through a fun house or its layout. Learning goals influenced study strategies and mem- 
ory. While learning, children with a layout goal spent more time in nonroute rooms and surveying the fun house perched atop a slide. When recalling, children with a layout goal remembered more relational information and more about nonroute rooms. In other words, they had better configural knowledge than children with a route goal. In a similar study with adults, Magliano, Cohen, Allen, and Rodrigue (1995) found that both source information and goal-driven processing influenced spatial memory. Groups with landmark, route, and configuration goals showed equal knowledge of landmarks and routes. Those with a configuration goal, however, provided more configural information in recall than did the other groups.

Although these studies indicate that navigational goals influence memory, the effects of goals on map learning have not been studied. Information on a map differs from that available during navigation in significant ways. In maps, individual landmarks lack distinction, inasmuch as they have symbolic representations, the most extreme case being a labeled point. Information in a map is twodimensional and not sequential, so that relative location and configural information are directly available. These differences suggest that map does learning follows a different course than does learning by navigation. In particular, in map learning, configural information is primary, whereas both landmark and route information are secondary, although available. Goals may direct attention to either primary or secondary information. Survey goals would emphasize configural information. Route goals would act on the secondary route information in one of two possible ways. They may focus attention on route information and away from configural information. This would predict better performance on route perspective tasks at the cost of survey perspective tasks. Alternatively, if the primacy of configural information is strong, route goals may serve a supplemental role, so that both configural and route information would be represented. This would predict no difference on survey perspective tasks but better performance on route perspective tasks.

\section{EXPERIMENT 1 Learning Method and Spatial Goal}

Method
Subjects
Fifty-two undergraduates from Tufts University participated in-
dividually in partial fulfillment of course credit. Approximately
even numbers of males and females were in each condition. The
subjects had not taken a class in the building used for this study.
Materials
Study materials. The first floor of the Psychology Research
Building, located on the Tufts University campus, served as the
stimulus environment. This building was chosen for two reasons:
The floor plan is complicated, and students have had little experi-
ence in the building, since it is not the primary Psychology De-
partment building since. This floor of the building consists of 23
individual rooms and connecting hallways. To reduce memory

load, we divided the building into 13 individual rooms and three complexes (combinations of 2 to 4 rooms in close proximity). All locations were designated by color names (e.g., Silver Room, Yellow Complex, etc.). The subjects either navigated through the building or studied a map (see Figure 1). For the navigation condition, signs indicating each location hung on the door(s). Plain white paper covered all other identifying signs, such as room numbers and faculty nameplates. The subjects could enter some rooms, such as those serving as classrooms, but could not enter others, such as faculty offices. The map of the building was printed on a standard $8.5 \times 11$ sheet of paper.

Each of the four conditions had individual instructions combining elements related to learning condition (map or navigation) and spatial goal (survey or route). For the learning condition, map instructions explained that a map of a building would be studied; navigation instructions explained that subjects would explore the first floor of the building on their own, entering open but not closed doors. For the spatial goal, the survey goal instructions asked the subjects to learn the layout of the building, whereas the route goal instructions asked them to learn the fastest routes between rooms and complexes. All the instructions informed the subjects of the minimum (10 $\mathrm{min}$ ) and a maximum (20 min) study time.

Test materials. Test materials consisted of three pairs of memory tasks assessing different aspects of spatial knowledge, including distance information, relative knowledge of two locations, and relative knowledge of three locations. Within each pair, one test assessed route knowledge, and the other assessed survey knowledge. Because of constraints on using the building, such as faculty privacy and equipment security, the subjects in the navigation conditions could not enter all rooms, giving them somewhat less knowledge about the size and extent of these rooms. To account for this, tasks assessed only information directly available to all the subjects. For example, because navigation subjects could not enter the Orange Room, test items referred to the Orange Room doorway, a location known to all the subjects.

The first pair of tasks required the subjects to estimate distances between two locations. For the route distance estimation task, a standard distance of 10 units (described as the distance between the Silver Room and the Maroon Room) was used to estimate route distances between 10 pairs of locations. For the Euclidean distance estimation task, a line representing the longest straight-line distance in the building (from the center of the Silver Room to the Orange Room doorway) served as the standard for each of 10 responses. The subjects marked their estimates on the standard line. Because distance estimates between two locations may be asymmetric if one is more salient (A. Tversky, 1977) or if a route is learned in one direction (Hazen, Lockman, \& Pick, 1978), half of the subjects received the pairs of locations in one order and half received the pairs in the reverse order for both of these tasks.

The second pair of tasks required the subjects to provide relative information about two locations. For the task requiring route knowledge, the subjects provided written descriptions of routes between 10 pairs of locations (route description task). For the task requiring survey knowledge, a walk-through-the-wall task was used. The four sides of the building were assigned direction terms (FUB, NID, DAL, and SOF). Nonsense names were used rather than canonical directions for two reasons. First, the building is not canonically oriented (the back wall faces northeast), so canonical terms could be confusing for subjects attuned to actual canonical directions. Second, canonical terms could bias use of a survey perspective. The nonsense direction terms were available to the subjects throughout the task. To complete the task, the subjects used a starting location and a designated direction to determine the correct adjacent location. For example, the subjects were asked, "Which room or complex would you be in if you walked through the DAL wall of the Maroon room?" The subjects repeated this 


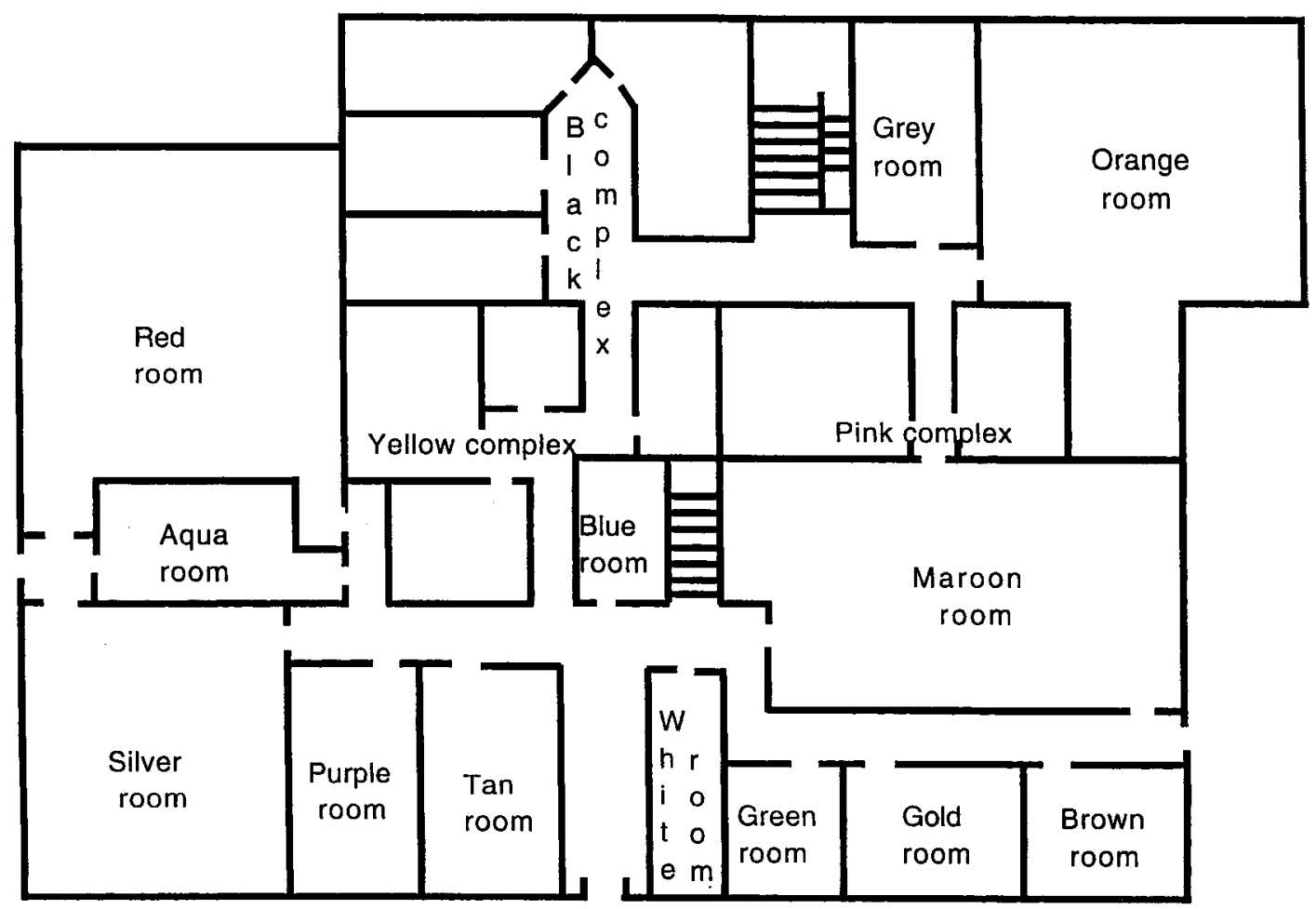

Figure 1. Map of Psychology Research Building, Tufts University.

task for 10 location-direction pairs. As with distance estimates, direction estimates are not always symmetric (Moar \& Bower, 1983), so for both tasks, half of the subjects received the pairs in one order, and half in the reverse order.

The third pair of tasks required the subjects to assess relative information about three locations. For the task requiring route knowledge, the subjects determined which two of three locations had the longest route distance between them (three-route task). The entire task consisted of 10 location triplets. For the task requiring survey knowledge, the subjects had to decide whether each of 10 configurations of three locations, designated by $\mathrm{Xs}$ and location labels, was accurate (spatial configuration task).

All the subjects received the distance estimation tasks, followed by the two-location tasks, followed by the three-location tasks. This order was believed to be the least biasing for later tasks. Within each pair, half of the subjects received the route knowledge task first, and half received the survey knowledge task first.

Individual difference materials. Individual difference materials consisted of a questionnaire and the Spatial Scanning 3 (SS3), Map Planning, Test from the Kit of Factor Referenced Cognitive Tests (Ekstrom, French, Harman, \& Dermen, 1995). The questionnaire asked the subjects to note their gender, handedness, familial sinistrality (FS + denotes subjects with positive familial sinistrality and FS - denotes subjects with no familial sinistrality), and preference for maps or verbal directions (referred to as preference).

\section{Design and Procedure}

The subjects were randomly assigned to one of the four experimental conditions. Navigation conditions were only run during evening and weekend hours, to avoid interference from other users of the building.
For the study phase of the experiment, the subjects first read the appropriate instructions for their condition. Then they studied the building, either by navigating or studying a map, for a minimum of $10 \mathrm{~min}$ and a maximum of 20 . An experimenter informed the subjects when the 10 -min minimum had elapsed. The subjects could self-terminate study at any point between 10 and $20 \mathrm{~min}$. The minimum study time provided some equivalence between the two learning conditions. Leiser et al. (1987) found that some of the difference in spatial memory based on simulated navigation and map study may be accounted for by differences in study time.

The subjects then worked through a packet of test materials at their own pace but were not allowed to return to a task to change responses. Finally, the subjects worked through the individual difference materials.

\section{Results}

Analyses consisted of analyses of variance (ANOVAs) with learning method (map or navigation) and spatial goal (survey or route) as between-subjects factors. Although not the focus of this research, individual differences on spatial tasks have previously received a great deal of interest. Consequently, additional analyses using gender, preferences for type of spatial information, and familial sinistrality as additional grouping factors are reported at the end of the results section.

No differences in study time emerged between the groups (map-survey goal, $M=10.40, S D=1.27$; maproute goal, $M=11.44, S D=2.83$; navigation-survey 
Table 2

Experiment 1: Memory Task Results, Means (With Standard Deviations)

\begin{tabular}{lll} 
& \multicolumn{2}{c}{ Spatial Goal } \\
\cline { 3 - 3 } \multicolumn{1}{c}{ Tasks } & \multicolumn{1}{c}{ Survey } & Route \\
\hline $\begin{array}{l}\text { Route distance estimations } \\
\text { Map }\end{array}$ & $6.62(1.32)$ units error & $5.22(2.08)$ units error \\
$\quad$ Navigation & $6.31(2.48)$ units error & $4.91(2.00)$ units error \\
Route descriptions & & \\
$\quad$ Map & $4.69(1.55)$ correct & $6.54(1.90)$ correct \\
$\quad$ Navigation & $7.69(1.03)$ correct & $8.23(1.16)$ correct \\
Three-route & & \\
$\quad$ Map & $5.15(2.04)$ correct & $6.46(1.61)$ correct \\
$\quad$ Navigation & $6.77(1.74)$ correct & $7.31(2.63)$ correct \\
Euclidean distance estimations & & \\
$\quad$ Map & $4.54(1.60)$ units error & $5.80(2.24)$ units error \\
$\quad$ Navigation & $7.78(2.09)$ units error & $7.78(3.32)$ units error \\
Walk-through-the-wall & & \\
$\quad$ Map & & \\
$\quad$ Navigation & $0.56(0.54)$ rooms off & $0.94(0.72)$ rooms off \\
Configuration & $1.28(0.33)$ rooms off & $1.07(0.75)$ rooms off \\
$\quad$ Map & & \\
$\quad$ Navigation & $7.08(1.71)$ correct & $7.00(2.38)$ correct \\
\hline
\end{tabular}

goal, $M=11.15, S D=1.74$; navigation-route goal, $M=$ $10.84, S D=1.81$ ).

\section{Spatial Memory Tests}

Table 2 presents the means and standard deviations for all the memory tasks. For those tasks indicating a number correct, there were 10 possible correct responses.

Tasks assessing route perspective knowledge. For the route distance estimation task, the average estimation error, using standardized units, was calculated for each subject. The results showed a significant effect of spatial goal $\left[F(1,48)=4.43, M S_{\mathrm{e}}=4.05, p<.05\right]$. The subjects who had a route goal ( $M=5.06$ units error) were more accurate than those who had a survey goal $(M=6.24)$. The mean correct estimation equaled 13.15 units, with a range from 5 to 22 units. For the route description task, each description was coded for accuracy. If the information provided by the subject accurately related the route, the description was coded as correct. This analysis also showed a significant effect of spatial goal $[F(1,48)=$ $\left.8.78, M S_{\mathrm{e}}=2.11, p<.01\right]$. The subjects given a route goal provided a greater number of correct descriptions than did subjects given a survey goal. Analyses also indicated a significant effect of learning condition $[F(1,48)=$ $\left.33.98, M S_{\mathrm{e}}=2.11, p<.001\right]$. The subjects who learned by navigating provided a greater number of correct descriptions than did those who studied a map. The interaction between spatial goal and learning condition was not significant. For the three-route task, responses were scored for accuracy. Results showed a significant effect of learning condition $\left[F(1,48)=4.73, M S_{\mathrm{e}}=4.17, p<\right.$ $.05]$. The subjects who learned by navigation $(M=7.04)$ provided more correct responses than did those who learned by studying a map $(M=5.81)$.
Tasks assessing survey perspective knowledge. For the Euclidean distance estimation task, the average estimation error was calculated for each subject. The results showed a significant effect of learning condition $[F(1,48)=$ $\left.15.46, M S_{\mathrm{e}}=5.73, p<.001\right]$. The subjects who studied a map $(M=5.17)$ gave more accurate estimates than did those who learned through navigation $(M=7.78)$. The mean correct estimation equaled 23.55 units, with a range of between 12.5 and 34.44 units. For the walkthrough-the-wall task, the subjects' responses were scored for the average number of rooms away from the correct response, with correct responses receiving a score of zero. The results showed a significant effect of learning condition $\left[F(1,48)=6.478, M S_{\mathrm{e}}=0.37, p<.05\right]$. Responses by subjects who studied maps $(M=0.75$ rooms off) were more accurate than those by navigation subjects $(M=1.18)$. For the spatial configuration task, responses were scored for the total number correct. No significant effects were found for performance on this task.

\section{Individual Differences}

Additional analyses assessed the role of individual differences, including gender, familial sinistrality, performance on the ETS Cognitive Battery test, and preference for type of spatial information. We had too few lefthanded subjects to allow analyses of handedness. Analyses assessing gender found no significant effects.

These analyses showed some main effects of the individual difference measures. With the exception of the preference of type of spatial information, each measure showed effects only on a few of the tasks, and no consistent pattern emerged. Main effect findings of preference showed better performance by individuals preferring maps. Of greater interest were findings showing interac- 
tions between preference and spatial goal. This interaction was seen for three of the six memory tasks-route distance estimations $[F(1,44)=3.81, p=.08]$, route descriptions $[F(1,44)=7.03, p<.05]$, and the spatial configuration task $[F(1,44)=5.72, p<.05]$. The interaction between preference and goal showed that the subjects who preferred maps and had a route goal performed best. The subjects preferring verbal directions showed little effect of goal.

\section{Discussion}

Both the spatial goal and the learning condition influenced performance on the spatial memory tasks. Spatial goals influenced performance on two of the three route perspective tasks. For route distance estimations and route descriptions, route goals led to better performance, regardless of learning condition. Analyses examining map learning and navigation separately, although not significant, showed the same pattern for each learning condition. This finding is somewhat consistent with the work of both Gauvain and Rogoff (1986) and Magliano et al. (1995). Their studies showed that a subject's spatial goal influenced both study strategies during navigation and memory for the environment. In particular, having a configuration goal led to better configural knowledge. Our study showed effects of having a route perspective goal but did not show corresponding effects for survey goals. Our findings also extend the role of goals to map learning, showing that map learners with a route goal performed better on route perspective tasks.

On the basis of the learning condition, the subjects' performance differed on two of the three route perspective tasks and on two of the three survey perspective tasks. The subjects provided more accurate route descriptions and responses on the three-route task if they had navigated. On the other hand, the subjects gave more accurate Euclidean distance estimates and responses to the walk-through-thewall task if they had studied a map. These results are consistent with others showing that spatial perspective is maintained in memory (Evans \& Pezdek, 1980; Sholl, 1987; Thorndyke \& Hayes-Roth, 1982).

Taken together, these results suggest that the spatial information source is predictive of the perspective represented in memory but is not the only predictor. Goals for using spatial information also influence perspective in memory. For navigation, the route goal enhanced readily available information. For map learning, the route goal seemed to play a supplemental role in guiding attention. The subjects who studied a map did well on survey perspective tasks, regardless of spatial goal. The subjects who studied a map and had a route goal also did better on route perspective tasks.

There are several explanations for why survey goals did not seem to influence performance. First, integrating knowledge gained through navigation may have been too difficult, consequently negating the impact of the survey goal. Learning environments by navigating is memory intensive, inasmuch as the amount of available information is limited to the visual field. New information taken in perceptually must be integrated with information held in memory. This is particularly true in an indoor environment, where walls limit the available information extensively. Second, it may take more time than was allotted in this study to develop a survey representation on the basis of navigation. When we walk through an environment, we perceptually update our route perspective knowledge. Forming and updating configural knowledge requires more effort. Thorndyke and HayesRoth (1982) found evidence that survey representations emerged from navigation, but only after extended exposure. Third, the subjects may have interpreted the survey goal differently, depending on the learning condition. The instructions for the survey goal informed the subjects that they were to learn the layout of the building. The subjects who navigated may have emphasized the layout of the routes and hallways in the building, whereas the subjects who studied a map may have emphasized the overall layout. The instructions for the route goal, to learn the fastest routes between locations, were fairly unambiguous. In Experiment 2, these explanations will be examined.

\section{EXPERIMENT 2 Specific Goal Instantiation}

The lack of any effect for the survey goal is examined in Experiment 2 by altering how the spatial goals are implemented by the subjects. Here, the subjects are given specific goal-related tasks to carry out while learning the environment. These tasks expand on one particular aspect of spatial perspective, the reference system. For a survey perspective, new landmarks are related to the known position of old landmarks. Therefore, the survey goal task involved determining all adjacent locations to each location in a specified set. For a route perspective, new landmarks are related to an egocentric position along a route. The route goal task involved determining the fastest routes an individual could take between a specified set of locations. The explicit study tasks focus on a particular element of each perspective, rather than on the perspectives in a more general sense. If the generality of the survey goal was problematic in Experiment 1 , we expect, on the basis of findings showing memory improvement when individuals pay attention to particular spatial information (Waddell \& Rogoff, 1987), to see goal effects on survey tasks in Experiment 2.

In contrast, if the difficulty of forming a survey representation on the basis of navigation is too high or if there is not enough time to form a survey representation, having a survey goal may not facilitate performance on survey tasks. Although some studies have found evidence that representations become more survey- or map- 
like over time (Thorndyke \& Hayes-Roth, 1982), this may occur only with extensive involvement in an environment. The subjects in both Experiment 1 and this study have limited experience in the environment, a maximum of $20 \mathrm{~min}$. If this were the case, we would expect to replicate the findings of Experiment 1, finding no goal effects for survey perspective tasks.

\section{Method}

\section{Subjects}

Sixty-six undergraduates from Tufts University participated individually for course credit. Approximately even numbers of males and females were in each condition. Two subjects were eliminated from analyses for failure to follow directions. The subjects were unfamiliar with the stimulus building.

\section{Materials}

The materials used in this experiment were the same as those in Experiment 1, with the following exceptions. Study instructions related to the spatial goals differed. For the route goal, instructions explained that the subjects would use a study list containing 10 pairs of location. While studying, they were to determine the fastest route between a pair of locations and then check off the pair on the study list, working through the entire list. For the survey goal, instructions explained that the subjects would use a study list of 10 locations. For each location, they were to determine which rooms and complexes were adjacent to this location and then check it off on the study list. No other written information could be added to the study lists.

Additional changes included the elimination of the ETS test and the addition of a survey task, map drawing. For the map drawing task, the subjects received a blank outline of the building and filled in the map, including room walls and labels. This task was added as a potentially more sensitive measure of survey perspective knowledge.

\section{Design and Procedure}

Procedure and design followed those used in Experiment 1.

\section{Results}

Task scoring and analyses were the same as those conducted for Experiment 1. Analysis of study time showed an effect of spatial goal $\left[F(1,60)=7.88, M S_{\mathrm{e}}=7.56\right.$, $p<.01]$. The subjects with a route goal $(M=13.76)$ studied longer than those with a survey goal $(M=11.83)$. No effect of learning condition emerged. Overall, the subjects' study time did not far exceed the minimum study time (map-survey goal, $M=12.36, S D=2.54$; map-route goal, $M=14.18, S D=3.41$; navigationsurvey goal, $M=11.31, S D=1.62$; navigation-route goal, $M=13.35, S D=3.09$ ).

\section{Spatial Memory Tests}

Table 3 presents means and standard deviations for all memory tasks. For tasks indicating a number correct, there are 10 possible correct responses.

Tasks assessing route perspective knowledge. One subject provided an outlier estimate of route distance, more than 10 times the average, and was eliminated from this analysis. This subject's performance on other tasks fell within the average response range. The results of the route distance estimation task showed no significant effects. Route descriptions were coded for accuracy, as in Experiment 1. Analysis of route description accuracy showed a main effect of learning condition $[F(1,60)=$ $\left.7.010, M S_{\mathrm{e}}=6.02, p<.05\right]$. The subjects who learned by navigation $(M=6.69)$ provided a greater number of accurate descriptions than did the subjects who studied maps $(M=5.06)$. No significant effects were found for performance on the three-route task.

Tasks assessing survey knowledge. Results of Euclidean distance estimations indicated a significant effect of learning condition $\left[F(1,60)=6.592, M S_{\mathrm{e}}=61.96, p<\right.$

Table 3

Experiment 2: Memory Task Results, Means (With Standard Deviations)

\begin{tabular}{|c|c|c|}
\hline \multirow[b]{2}{*}{ Tasks } & \multicolumn{2}{|c|}{ Spatial Goal } \\
\hline & Survey & Route \\
\hline \multicolumn{3}{|c|}{ Route distance estimations } \\
\hline Map & $6.64(1.58)$ units error & $5.37(1.92)$ units error \\
\hline Navigation & $5.11(1.91)$ units error & $5.61(2.23)$ units error \\
\hline \multicolumn{3}{|l|}{ Route descriptions } \\
\hline Map & $5.38(1.89)$ correct & $4.75(2.84)$ correct \\
\hline Navigation & $6.81(2.17)$ correct & $6.56(2.78)$ correct \\
\hline \multicolumn{3}{|l|}{ Three-route } \\
\hline Map & $6.69(2.24)$ correct & $6.19(2.23)$ correct \\
\hline Navigation & $6.38(2.09)$ correct & $7.25(2.08)$ correct \\
\hline \multicolumn{3}{|c|}{ Euclidean distance estimations } \\
\hline Map & $5.71(2.52)$ units error & $6.02(2.16)$ units error \\
\hline Navigation & $7.73(2.18)$ units error & $6.81(1.83)$ units error \\
\hline \multicolumn{3}{|c|}{ Walk-through-the-wall } \\
\hline Map & $0.29(0.65)$ rooms off & $0.94(0.80)$ rooms off \\
\hline Navigation & $0.71(0.54)$ rooms off & $0.86(0.59)$ rooms off \\
\hline \multicolumn{3}{|l|}{ Configuration } \\
\hline Map & $7.69(1.20)$ correct & $7.25(2.18)$ correct \\
\hline Navigation & $6.69(2.30)$ correct & $6.63(2.16)$ correct \\
\hline \multicolumn{3}{|l|}{ Map drawing } \\
\hline Map & $0.96(0.11)$ proportion correct & $0.76(0.23)$ proportion correct \\
\hline Navigation & $0.79(0.25)$ proportion correct & $0.71(0.22)$ proportion correct \\
\hline
\end{tabular}


$.05]$. The subjects who studied maps $(M=5.87)$ gave more accurate estimates than did those who learned by navigating $(M=7.27)$. The walk-through-the-wall task showed a main effect of spatial goal $[F(1,60)=6.049$, $\left.M S_{\mathrm{e}}=0.423, p=.05\right]$. The subjects who had a survey goal ( $M=0.50$, rooms off) were closer to the correct response than were those who had a route goal $(M=0.90)$. No significant effects were found for performance on the spatial configuration task.

Map drawings were coded for accuracy. For each location on the map, the proportion of rooms in the correct relative location was determined. A room was considered in the correct location if adjacency, within $2.5 \mathrm{in}$., and directionality were maintained. The criterion of 2.5 in. equals approximately one and a half times the average height and width of the rooms on the map and takes into account distance for hallways. The average proportion for each subject was then arcsine transformed and submitted to analysis (for greater interpretability, untransformed mean proportions are reported). This analysis showed a significant main effect of spatial goal $\left[F(1,60)=9.349, M S_{\mathrm{e}}=0.137, p<.005\right]$. The subjects with a survey goal $(M=0.88)$ drew more rooms in correct location than did those with a route goal $(M=0.74)$. The analyses also showed a main effect of learning condition $\left[F(1,60)=5.072, M S_{\mathrm{e}}=0.137, p<.05\right]$. The subjects who studied maps $(M=0.86)$ drew more rooms in the correct location than did those who learned by navigating $(M=0.75)$. No interaction between spatial goal and learning condition emerged.

Individual differences. Individual difference measures included gender, preference, and familial sinistrality. Analyses showed interactions of individual difference

Table 4

Experiment 2: Interactions Between Learning Conditions and Familial Sinistrality

\begin{tabular}{lll}
\hline & \multicolumn{2}{c}{ Familial Sinistrality } \\
\cline { 2 - 3 } Tasks & Left Handers & No Left Handers \\
\hline $\begin{array}{l}\text { Route distance estimations } \\
\quad \text { Map }\end{array}$ & 6.72 units error & 5.78 units error \\
$\quad$ Navigation & 4.48 units error & 5.66 units error \\
Route descriptions & & \\
$\quad$ Map & 4.25 correct & 5.33 correct \\
$\quad$ Navigation & 8.25 correct & 6.17 correct \\
Three-route & & \\
$\quad$ Map & 6.12 correct & 6.54 correct \\
$\quad$ Navigation & 8.12 correct & 6.38 correct \\
Walk-through-the-wall & & \\
$\quad$ Map & 0.85 rooms off & 0.53 rooms off \\
$\quad$ Navigation & 0.26 rooms off & 0.96 rooms off \\
Spatial configuration & & \\
$\quad$ Map & 7.00 correct & 7.62 correct \\
Navigation & 7.75 correct & 6.29 correct \\
Map drawing & & \\
$\quad$ Map & $78.5 \%$ correct & $88.8 \%$ correct \\
Navigation & $92.0 \%$ correct & $69.8 \%$ correct \\
\hline
\end{tabular}

Note-Units are indicated, as some tasks examined error and others examined accuracy. measures with both spatial goal and learning condition. Gender interacted with spatial goal on the route description task $\left[F(1,56)=5.565, M S_{\mathrm{e}}=5.716, p<.05\right]$. Females gave more correct descriptions when they had a survey goal; males gave more correct descriptions when they had a route goal. Familial sinistrality interacted with learning condition for six of the seven memory tasks. All interactions were significant at the .05 level. For route perspective tasks, FS + subjects performed better after navigating, whereas FS - subjects showed no difference on the basis of learning condition. For survey perspective tasks, FS + subjects showed better performance after navigating and FS - subjects performed better after studying maps (see Table 4).

Analyses based on subjects' preference showed main effects for six of the seven memory tasks. All effects were significant at the .05 level. In all cases, the subjects preferring maps outperformed those preferring verbal descriptions.

\section{Discussion}

As in Experiment 1, both spatial goal and learning condition influenced performance. In contrast to Experiment 1 , the spatial goal influenced performance on survey perspective tasks, but not on route perspective tasks. This finding runs counter to the notion that survey perspective representations were too difficult to develop. In addition, the particular survey perspective tasks that showed effects of goal support the notion that the survey goal instructions from Experiment 1 may have had alternate interpretations. The survey goal study task in this experiment directed attention to relative spatial information. The two survey perspective tasks showing significant goal effects make the most use of the adjacency information emphasized by the study task, namely the walk-through-the-wall task and map drawing.

In addition, the fact that these tasks, and not other survey perspective tasks, showed goal effects supports an attentional role in goal-directed processing (Gagne \& Rothkopf, 1975; Goetz, Schallert, Reynolds, \& Radin, 1983; Pichert \& Anderson, 1977; Rothkopf \& Billington, 1979). If goal-directed processing affected schema instantiation, effects should have been seen for other survey tasks, indicating a broader survey perspective schema.

Surprisingly, this experiment did not show effects of route perspective goals. In this case, the same factors that disambiguated the survey goal may have overspecified the route goal. The choice of room pairs for the study task was designed to ensure that the subjects experienced all rooms and corresponding routes. The subjects may have concentrated primarily on the end points of the routes, those included on the study list. If this was the case, much of the spatial learning may have been implicit. The memory tasks did not test the same pairs used in the study task. Waddell and Rogoff (1987) found that intentional learning of spatial information superseded implicit learning, even when implicit learning required 
attention to individual locations, such as rating the appropriateness of object positions.

As in Experiment 1, learning condition also influenced performance. The subjects performed better on tasks consistent with the perspective learned. Learning condition effects were evident on two of the three route perspective tasks and on three of the four survey perspective tasks.

\section{GENERAL DISCUSSION}

In two experiments, the subjects learned an environment either by studying a map or by navigating. During acquisition, the subjects had a spatial goal, either to learn the layout of the building (survey goal) or to learn the fastest routes between locations (route goal). Memory tasks assessed both route and survey perspective processing. In both experiments, performance differed as a result of both spatial goal and learning condition. More specifically, the relative salience of perspective information during acquisition was influenced by both how information was learned and why it was learned. Transfer appropriate processing (Morris et al., 1977) can be evoked in both cases. Given no goal, assumptions about testing rely heavily on the learning condition, making that perspective salient. With a goal, assumptions about testing take the goal into account. In both cases, acquisition is being matched to the assumed content of testing. Under this framework, findings showing maintenance of the learned perspective (e.g., Sholl, 1987; Thorndyke \& Hayes-Roth, 1982) and those showing a more flexible use of perspective (Taylor \& Tversky, 1992a, 1996) can begin to be reconciled.

Although equivocal, previous work has shown that the information source perspective is maintained in memory (Evans \& Pezdek, 1980; Leiser et al., 1987; Perrig \& Kintsch, 1985; Sholl, 1987; Thorndyke \& Hayes-Roth, 1982). Our studies include additional controls in addressing this issue. The same environment was used throughout, as environmental features change the probabilities of using a particular perspective (Taylor \& Tversky, 1996) and are subject to different types of cognitive preprocessing (B. Tversky, 1981). Length of exposure to the environment was controlled. Leiser et al. found that length of study time may account for performance differences based on map and navigation learning. Finally, actual, rather than simulated, navigation was used. Some forms of simulated navigation include elements of survey knowledge (Leiser et al., 1987). With our additional controls, both studies found that subjects who studied maps showed better survey knowledge and subjects who learned by navigation showed better route knowledge.

The learning condition is not the only factor influencing the mental representation of perspective. Spatial goals also played a role. Goals increase attention to goalrelevant information. Variability in subjects' goals in previous studies may have influenced their results. Some studies certainly had varied goals, as they used environ- ments with which the subjects had previous experience (Evans \& Pezdek, 1980; Sholl, 1987). Other studies included both implicit and explicit learning goals. Thorndyke and Hayes-Roth (1982) assumed that their navigation subjects learned the environment as employees in the building, whereas map subjects studied to successfully redraw the map, a survey perspective goal. Waddell and Rogoff (1987) have found differences in spatial memory on the basis of implicit versus explicit learning. All the subjects in our study had explicit learning goals.

Two studies have explicitly examined the role of goals during navigation (Gauvain \& Rogoff, 1986; Magliano et al., 1995). Our findings extended these by showing that goals influenced representations based on both navigation and map learning. Importantly, our findings indicate that spatial goals highlight perspective-relevant information, even information that is not most available during learning. In addition, these findings indicated that the representation of perspective is flexible to the extent that it can be changed through the course of purposeful study. Individuals learning by navigation can, even over a short learning interval, encode survey-type knowledge if goals direct attention to survey information. Likewise, route knowledge can be encoded during map study if goals direct attention to route information.

Not all memory tasks showed the effects of learning condition and spatial goal. The tasks were chosen to assess processing from a particular spatial perspective and have been used previously for this purpose. Even with careful selection, performance on tasks addressing a particular perspective were inconsistent. Additional analyses using correlation and factor analysis showed no consistent pattern on the basis of perspective. Task performance correlated both within and between perspectives, but not for all tasks. Factor analysis results showed that both route and survey perspective tasks would load on a single factor.

These findings bring up the question of what cognitive processes these tasks are recruiting - in particular, what cognitive processes, other than those related directly to spatial perspective, may be impacting performance? We know that distance estimation tasks are not immune to distortions. Route distance estimations are influenced by the number of landmarks along the route (McNamara, Ratcliff, \& McKoon, 1984), the number of turns in the route (Sadalla \& Magel, 1980), and the relative familiarity of landmarks (Briggs, 1973). Hierarchical grouping affects Euclidean distance estimations (McNamara et al., 1989). Individuals must be processing more than distance information when making these judgments. In our studies, the two distance estimation tasks showed different effects of spatial goal and learning condition, with one task showing a significant effect of spatial goal and the other an effect of learning condition, particularly in Experiment 1. Although more research needs to be done to definitively understand the processes underlying performance on these tasks, two possibilities exist to explain our results. First, these tasks may vary in their susceptibility to other ongoing cognitive processes, which 
could include goal processing. Second, these tasks may not be tapping the processing occurring during acquisition, the time when learning condition and spatial goal would have the greatest effect (Morris et al., 1977). Recognition, cued recall, and free recall all purportedly tap memory; however, many studies have shown that different acquisition tasks may lead to good performance on recognition tests but not on recall tests, or vice versa (e.g., B. Tversky, 1973).

Individual differences also contributed to performance variance. Although equivocal, theories of hemispheric lateralization have been implicated in differences based on gender, handedness, and familial sinistrality (Levy, 1969; McKeever, 1986; Snyder \& Harris, 1993). Interestingly, our studies showed that gender and familial sinistrality interacted with our independent variables, rather than showing main effects. In addition, individual differences were not evident for all tasks. Therefore, individual differences alone did not separate performance.

Self-reported preferences for maps or verbal directions showed fairly consistent individual differences. Individuals preferring maps performed better and were influenced to a greater degree by spatial goals. Self-report measures have been criticized as being unreliable indices of individual differences, but findings such as ours (see also Kozlowski \& Bryant, 1977) increase their validity. The direction of this effect, however, cannot be determined; preference for maps may be predictive of or may be a consequence of better spatial ability.

The results presented here indicate multiple influences on the representation of spatial perspective. The learned perspective does influence the perspective remembered. Spatial goals also influence spatial knowledge, regardless of the learning condition. Individual differences also played a role, but primarily in combination with learning condition and goal. Current and future studies in our laboratory will continue to address issues of spatial perspective. One pressing issue brought to light in these studies addresses the cognitive processes recruited by various tasks assessing perspective. Until we understand these tasks more fully, it is difficult to draw definitive conclusions about the representation of perspective.

Another issue is the degree to which information is symbolic. Map learning, as compared with navigation, requires the translation of symbols representing the environment. Text requires yet another level of translation. Studies showing flexibility in the representation of perspective have primarily used texts to present spatial information (Taylor \& Tversky, 1992b, 1995, 1996). The translation process may make certain spatial properties more salient, thus leading to greater flexibility in perspective. The translation process is also susceptible to other forms of cognitive preprocessing, such as grouping. In the process of grouping, the reliance on specific perspective information may decrease. The work presented here answers some questions about the representation of spatial perspective, while, at the same time, making apparent the complexity of the issue.

\section{REFERENCES}

Anderson, R. C., \& Pichert, J. W. (1978). Recall of previously unrecallable information following a shift in perspective. Journal of Verbal Learning \& Verbal Behavior, 17, 1-12.

BRIGGS, R. (1973). Urban cognitive distances. In R. Downs \& D. Stea (Eds.), Image and environment: Cognitive mapping and spatial behavior (pp. 361-388). Chicago: Aldine.

Britton, B. K., Meyer, B. J. F., Simpson, R., Holdredge, T. S., \& CURRY, C. (1979). Effects of the organization of text on memory: Tests of two implications of a selective attention hypothesis. Journal of Experimental Psychology: Human Learning \& Memory, 5, 496-506.

Ekstrom, R. B., French, J. W., harman, H. H., \& Dermen, D. (1995). Kit of factor-referenced cognitive tests. Princeton, $\mathrm{NJ}$ : Educational Testing Service.

Evans, G. W., Marrero, D. G., \& Butler, P. A. (1981). Environment learning and cognitive mapping. Environment \& Behavior, 13, 83104.

Evans, G. W., \& PeZdeK, K. (1980). Cognitive mapping: Knowledge of real-world distance and location information. Journal of Experimental Psychology: Human Learning \& Memory, 6, 13-24.

GAGNE, E. D., \& RoThKoPf, E. Z. (1975). Text organization and learning goals. Journal of Educational Psychology, 67, 445-450.

GaUVAIN, M., \& RogOFF, B. (1986). Influence of the goal on children's exploration and memory of large-scale space. Developmental Psychology, 22, 72-77.

Goetz, E. T., Schallert, D. L., Reynolds, R. E., \& Radin, D. I. (1983). Reading in perspective: What real cops and pretend burglars look for in a story. Journal of Educational Psychology, 75, 500-510.

Golledge, R. G., \& SPECTOR, N. A. (1978). Comprehending the urban environment: Theory and practice. Geographical Analysis, 14, 305325.

Hazen, N. L., Lockman, J. J., \& Pick, H. L. (1978). The development of children's representation of large-scale environments. Child Development, 49, 623-636.

KozLoWSKI, L. T., \& BRYANT, K. J. (1977). Sense of direction, spatial orientation, and cognitive maps. Journal of Experimental Psychology: Human Perception \& Performance, 3, 590-598.

KuIPERS, B. (1978). Modeling spatial knowledge. Cognitive Science, 2 , 129-153.

LABERGE, D. (1995). Attentional processing: The brain's art of mindfulness. Cambridge, MA: Harvard University Press.

Leiser, D., TZelgov, J., \& HenIK, A. (1987). A comparison of map study methods: Simulated travel vs. conventional study. Australian Journal of Psychology, 7, 317-334.

LEISER, D., \& ZilBershATZ, A. (1989). The Traveller: A computational model of spatial network learning. Environment \& Behavior, 21, 435463.

Levy, J. (1969). Possible basis for the evolution of lateral specialisation of the human brain. Nature, 224, 614-615.

Magliano, J. P., Cohen, R., Allen, G. L., \& Rodrigue, J. R. (1995). The impact of a wayfinder's goal on learning a new environment: Different types of spatial knowledge as goals. Journal of Environmental Psychology, 15, 65-75.

McDonald, T. P., \& Pellegrino, J. W. (1993). Psychological perspectives on spatial cognition. In T. Garling \& R. G. Golledge (Eds.), Behavior and environment: Psychological and geographical approaches (pp. 47-82). Amsterdam: Elsevier.

MCKeEVER, W. F. (1986). Tachistoscopic methods in neuropsychology. In H. J. Hannay (Ed.), Experimental techniques in human neuropsychology (pp. 167-211). New York: Oxford University Press.

MCNAmaRA, T. P. (1986). Mental representation of spatial relations. Cognitive Psychology, 18, 87-121.

MCNamara, T. P., Hardy, J. K., \& HirTle, S. C. (1989). Subjective hierarchies in spatial memory. Journal of Experimental Psychology: Learning, Memory, \& Cognition, 15, 211-227.

MCNamara, T. P., RatclifF, R., \& McKoon, G. (1984). The mental representation of knowledge acquired from maps. Journal of Experimental Psychology: Learning, Memory, \& Cognition, 10, 723-732. 
MEYER, B. J. F. (1975). The organization of prose and its effects on memory. Amsterdam: North-Holland.

MOAR, I., \& BOWER, G. H. (1983). Inconsistency in spatial knowledge. Memory \& Cognition, 11, 107-113.

MorRIS, C. D., Bransford, J. D., \& FranKs, J. J. (1977). Levels of processing versus transfer appropriate processing. Journal of Verbal Learning \& Verbal Behavior, 16, 519-533.

Perrig, W., \& KintsCh, W. (1985). Propositional and situational representations of text. Journal of Memory \& Language, 24, 503-518.

Pichert, J. W., \& ANderson, R. C. (1977). Taking different perspectives on a story. Journal of Educational Psychology, 69, 309-315.

RoThKOPF, E. Z., \& BilLington, M. J. (1979). Goal guided learning from text: Inferring a descriptive processing model from inspection times and eye movements. Journal of Educational Psychology, 71, 310-327.

Sadalla, E. K., \& MaGel, S. G. (1980). The perception of traversed distance. Environment \& Behavior, 12, 65-79.

SHolL, M. J. (1987). Cognitive maps as orienting schemata. Journal of Experimental Psychology: Learning, Memory, \& Cognition, 13, $615-$ 628.

SноLL, M. J. (1992, November). Frames of reference used in accessing spatial knowledge. Paper presented at the 33rd Annual Meeting of the Psychonomic Society, St. Louis.

SIEGEL, A. W., \& WhITE, S. H. (1975). The development of spatial representations of large-scale environments. In H. W. Reese (Ed.), $A d$ vances in child development and behavior (Vol. 10, pp. 9-55). New York: Academic Press.

SNYDER, P. J., \& HarRis, L. J. (1993). Handedness, sex, and familial sinistrality effects on spatial tasks. Cortex, 29, 115-134.
Stevens, A., \& Coupe, P. (1978). Distortions in judged spatial relations. Cognitive Psychology, 10, 422-437.

TAYLOR, H. A., \& TVERSKY, B. (1992a). Descriptions and depictions of environments. Memory \& Cognition, 20, 483-496.

TAYLOR, H. A., \& TVERSKY, B. (1992b). Spatial mental models derived from survey and route descriptions. Journal of Memory \& Language, 31, 261-292.

TAYLOR, H. A., \& TVERSKY, B. (1995). Assessing spatial representation using text. Geographical Systems, 2, 235-254.

TAYlor, H. A., \& TVersky, B. (1996). Perspective in spatial descriptions. Journal of Memory \& Language, 35, 371-391.

THORNDYKE, P. W., \& HAYEs-ROTH, B. (1982). Differences in spatial knowledge acquired from maps and navigation. Cognitive Psychology, 14, 560-589.

TVERSKY, A. (1977). Features of similarity. Psychological Review, 84 327-352.

TVERSKY, B. (1973). Encoding processes in recognition and recall. Cognitive Psychology, 5, 275-287.

TVerSKY, B. (1981). Distortions in memory for maps. Cognitive Psychology, 13, 407-433.

TVersky, B., \& SChiano, D. J. (1989). Perceptual and conceptual factors in distortions in memory for graphs and maps. Journal of Experimental Psychology: General, 118, 387-398.

WADDELL, K. J., \& RoGoFF, B. (1987). Contextual organization and intentionality in adults' spatial memory. Developmental Psychology, 23, 514-520.

(Manuscript received May 28, 1997; revision accepted for publication November 17, 1997.) 